\title{
Escala de Gestión del Aprendizaje Cooperativo en el Aula
}

\section{Learning Cooperation Management Scale in Classroom}

\author{
Pedro Antonio Sánchez-Miguel ${ }^{1}$, Santiago Mendo-Lázaro ${ }^{2}$, Benito León-Del Barco ${ }^{3}$, Diana Amado ${ }^{4}$ y \\ Damián Iglesias-Gallego ${ }^{5}$
}

\section{Resumen}

El objetivo de esta investigación fue construir y validar la escala de gestión de aprendizaje cooperativo en una muestra de maestros y profesores de enseñanza secundaria en España. La muestra estuvo formada por 376 maestros y profesores, pertenecientes a enseñanza infantil $(n=136)$, primaria $(n=159)$ y secundaria $(n=71)$, tanto de género masculino $(n=110)$ como femenino $(n=266)$, y de centros públicos $(n=269)$, concertados $(n=106)$ y privados $(n=1)$ de la Región de Extremadura. Los resultados obtenidos mostraron que el instrumento presenta una adecuada validez factorial y consistencia interna, mediante la generación de 3 factores: Diseño, Seguimiento y Evaluación. Finalmente, se concluye que este trabajo aporta evidencias científicas de que la escala de gestión de aprendizaje cooperativo se muestra válida y fiable para analizar esas estrategias a desarrollar en el aula en profesores y maestros españoles.

Palabras clave: maestros, enseñanza, aprendizaje cooperativo, propiedades psicométricas

\begin{abstract}
The aim of this research was to validate the management of cooperative learning scale in a sample size of teachers from Primary and Secondary schools in Spain. The sample was composed of 376 teachers belonged to Childhood $(n=136)$, Primary $(n=159)$ and Secondary $(n=71)$ schools, both male $(n=110)$ and female $(n=266)$, from public $(n=269)$, state $(n=106)$ and private $(n=1)$ schools from the Region of Extremadura. Results showed that the instrument had an adequate factorial validity and internal consistency, revealing a structure of 3 factors: Design, Follow-Up and Evaluation. Finally, this work gives empiric evidences that the management of cooperative learning scale is valid and reliable to examine these behaviors in Spanish teachers.
\end{abstract}

Keywords: teachers, teaching, cooperative learning, psychometrics properties

\footnotetext{
${ }^{1}$ Doctor en Ciencias del Deporte. Departamento de Didáctica de la Expresión Musical, Plástica y Corporal. Facultad de Formación del Profesorado. Universidad de Extremadura. Avenida de la Universidad, S/N, 10071. Cáceres, España. Tel.: 927257049. Correo: pesanchezm@unex.es; diglesia@unex.es (correspondencia)

${ }^{2}$ Doctor en Psicología. Departamento de Psicología y Antropología. Facultad de Formación del Profesorado. Universidad de Extremadura. Avenida de la Universidad, S/N, 10071. Cáceres, España. Correo: smendo@unex.es

${ }^{3}$ Doctor en Psicología. Departamento de Psicología y Antropología. Facultad de Formación del Profesorado. Universidad de Extremadura. Avenida de la Universidad, S/N, 10071. Cáceres, España. Correo: bleon@unex.es

${ }^{4}$ Doctora en Ciencias del Deporte. Centro de Estudios del Deporte. Universidad Rey Juan Carlos. Área de Educación física. Avda. de Atenas s/n 28922, Alcorcón, Madrid, España. Correo: diana.amado@urjc.es

${ }^{5}$ Doctor en Ciencias del Deporte. Departamento de Didáctica de la Expresión Musical, Plástica y Corporal. Facultad de Formación del Profesorado. Universidad de Extremadura. Avenida de la Universidad, S/N, 10071. Cáceres, España. Correo: diglesia@unex.es
} 


\section{Introducción}

El desarrollo de habilidades sociales, de cooperación y colaboración son cada vez más valiosas en entornos sociales y profesionales (OECD, 2015). Estas habilidades no son ajenas a contextos laborales como el contexto educativo, que requiere de profesionales que adapten y conozcan nuevos conocimientos y destrezas, y donde se ha demostrado que la capacidad de fomentar conductas cooperativas en el alumnado, conlleva la aparición de consecuencias positivas como la mejora en el aprendizaje, mejoras sociales y afectivas (León, Mendo, Felipe, Polo, \& Fajardo, 2017; Slavin, 2011). De hecho, se trata de una innovación educativa que trata de resolver problemas tan graves como el bullying, el fracaso escolar, la discriminación, la xenofobia, intentando mejorar la relación entre profesores y alumnos y la relación entre iguales. Además, combina el desarrollo de competencias intelectuales y profesionales, el desarrollo de estrategias de comunicación y el crecimiento personal del alumno (León, Felipe, Iglesias, \& Latas, 2011).

En este sentido, el aprendizaje cooperativo (AC) se ha mostrado como una herramienta a aplicar en el contexto educativo de máximo interés, debido a sus múltiples implicaciones en la formación integral del alumnado. El AC se define como una metodología de aprendizaje basada en el estudiante, que aplicándose de forma correcta puede promover y favorecer competencias y destrezas útiles para su desarrollo profesional y su vida social (Atxurra, Villardón-Gallego, \& Calvete, 2015). Este modelo pedagógico e ha estudiado desde hace décadas y desde diversas concepciones teóricas (ver Slavin, 2011, para revisión teórica).

Así, desde la concepción de psicología social, en el AC un individuo alcanza su objetivo si los otros miembros alcanzan el suyo (Deutsch, 1949). Desde la concepción conductista, en el AC las recompensas o refuerzos del individuo son directamente proporcionales a la calidad del trabajo en grupo (Kelley \& Thibaut, 1969). Para otros autores como Johnson y Johnson (2009), el AC está basado en grupos de trabajo que son estructurados por los profesores con el objetivo de maximizar el aprendizaje social, la motivación y los resultados a nivel cognitivo.

En base a estas concepciones, el AC se considera que está compuesto por cinco factores clave: interdependencia positiva, interacción, compromiso individual, enseñanza de destrezas interpersonales y sociales, y calidad de los procesos de grupo. Las situaciones de aprendizaje no son cooperativas si los estudiantes no están organizados en grupos donde se muestre una interdependencia positiva y donde se favorezca la cohesión de grupo como medio para alcanzar los objetivos grupales (Johnson \& Johnson, 2009). La responsabilidad individual hace referencia a la capacidad del individuo de dar lo mejor de sí, presentar sus ideas al grupo, mostrarse disponible en el aprendizaje de tareas... (Gorostiaga, Balluerka, Ulacia, \& Aliri, 2018; Johnson, 2009).

Por otro lado, el compromiso individual se muestra como la capacidad que tienen los individuos de generar fuerzas que permitan compartir y fomentar el trabajo de los otros miembros del grupo (Johnson \& Johnson, 2005). Las destrezas sociales (i.e. prestar atención, respecto por las opiniones de los otros, plantear preguntas de forma conjunta, etc.) nos permiten trabajar de forma eficiente y eficaz en el desarrollo de tareas, mientras que la calidad en los procesos de grupo hace referencia a la capacidad para coordinar esfuerzos y para conseguir objetivos comunes, mediante la confianza entre los miembros, la comunicación adecuada, la aceptación de cada miembro del grupo, y la capacidad de resolver los problemas de forma constructiva (Johnson \& Johnson, 2009).

La formación en AC por parte de los docentes se muestra crucial, independientemente del nivel educativo o titulación en la que se encuentre el alumnado (Biggs \& Tang, 2011; Cavanagh, 2011). En este sentido, Hattie (2008) demostró en su meta-análisis los mejores y mayores beneficios académicos de este modelo en comparación con aquellos individualistas o competitivos, tanto en la educación primaria o secundaria (Gillies \& Boyle, 2010) como universitaria (Johnson \& Johnson, 2002).

No obstante, en estudios anteriores (Gilies \& Boyle, 2006) se destaca la falta de preparación en el diseño y seguimiento del AC, como uno de los factores que incidían en mayor medida para no 
implementar este modelo didáctico en las aulas. Además, un problema que se encontraban muchos docentes al aplicar el AC en el contexto educativo, es la forma de evaluar las estrategias y recursos que se desarrollan durante las clases por el docente (Legrain, Escalé, Lafont, \& Chaliès, 2018).

Por tanto, es importante llevar a cabo una adecuada organización de los grupos de trabajo y gestión de los recursos (Dyson \& Casey, 2016) y hacer un seguimiento en las actividades y tareas para promover la interacción entre los estudiantes (Johnson \& Johnson, 2009). Además, mediante una evaluación correcta podremos conocer si se desarrolla de una manera adecuada la responsabilidad individual en el alumnado (Gorostiaga et al., 2018; Johnson, 2009).

Por lo tanto, y teniendo en cuenta lo expuesto anteriormente, se muestra el AC como una de las formas de pedagogía activa más utilizadas, donde el alumnado interacciona con sus compañeros y el contexto que lo rodea (Adams \& Hamm, 1994). Dentro de ese contexto, el docente ocupa un lugar destacado en el diseño y evaluación de las tareas, así como en el seguimiento de las mismas. Hasta el momento, las escalas que se han empleado para analizar el AC en el contexto educativo han tratado de analizar la interdependencia positiva, interacción, habilidades sociales, reflexión grupal, hetereogeneidad, evaluación y tutoría, como la diseñada por Atxurra, Villardón-Gallego, y Calvete (2015) para conocer el grado de aplicación del aprendizaje cooperativo en la universidad con una muestra de 1460 estudiantes de las Universidades de Deusto (España) y la Universidad Católica de Temuco (Chile), mostrando un instrumento válido y fiable para valorar el aprendizaje cooperativo en el aula.

Anteriormente, Johnson y Johnson (1983), ya habían utilizado alguno de esos factores como la interdependencia positiva, evaluación y tutorías para elaborar el Classroom Life Instrument (CLI). Sin embargo, este instrumento no contenía elementos claves que definen el aprendizaje cooperativo como la reflexión grupal, interacción o habilidades sociales, que reflejan las escalas posteriores.

En la misma línea, Fernández-Río, Cecchini, Méndez-Giménez, Méndez-Alonso y Prieto (2017) validaron una escala de aprendizaje cooperativo formada por interacción promotora, interdependencia positiva, responsabilidad individual, procesamiento grupal y habilidades sociales, en el contexto educativo en estudiantes de primaria, secundaria y bachillerato de 68 centros educativos de toda España, mostrando una adecuada validez convergente, discriminante y concurrente.

Por tanto, la investigación sobre $\mathrm{AC}$ se ha centrado fundamentalmente en conocer las relaciones y el resultado de su aplicación sobre aspectos académicos (Dat Tran, 2014), sociales (Monereo, Castillo, \& Martínez-Fernández, 2013) y afectivos (Yi \& LuXi, 2012), o en conocer la opinión del alumnado respecto a la metodología cooperativa, siendo pocos los trabajos científicos que han tratado de analizar el aprendizaje cooperativo desde la perspectiva del profesorado, así como de los mecanismos mediadores implicados en la metodología de aprendizaje cooperativo (León, Felipe, Iglesias, \& Marugán, 2014), como puede ser el rol del docente en el AC.

De esta manera, tal y como se ha indicado previamente, se ha mostrado que la gestión, seguimiento y evaluación del AC por parte de los docentes es esencial para el éxito de las estructuras cooperativas en el aula. El docente debe tomar estructurar y decisiones antes de abordar la enseñanza, explicar a los estudiantes la tarea, orientar y supervisar a los equipos, evaluar el nivel de aprendizaje de los alumnos y alentarlos a determinar con qué eficacia están funcionando sus grupos de aprendizaje (Johnson, Johnson, \& Holubec, 1999; Salavera \& Usán, 2018).

En este sentido, y siguiendo los hallazgos de la literatura científica, es de gran interés conocer el grado en que los dominios de diseño, evaluación y seguimiento de las actividades cooperativas en un instrumento nuevo, y comprobar si el grupo de ítems es adecuado para la evaluación del constructo, indicando y demostrando el grado de coherencia con el marco conceptual indicado previamente, y si los procesos llevados a cabo son adecuados, garantizado por la correcta utilización de los criterios metodológicos (Grapentine, 1995).

Así, y teniendo en cuenta este último aspecto, el objetivo de este trabajo es construir y validar la escala de gestión del aprendizaje cooperativo en el aula en una muestra de maestros de infantil y 
primaria, y profesores de enseñanza secundaria en España. De la formulación de este objetivo, se plantea la hipótesis de que la validación de la escala de gestión y cooperación en el aula presentará una adecuada validez y fiabilidad en una muestra de maestros y profesores españoles. Además, se plantea como hipótesis la relación positiva de los constructos de la escala de aprendizaje cooperativo en el aula con la Escala de Compromiso profesional y aspiraciones de desarrollo profesional.

\section{Método}

\section{Participantes}

La muestra estuvo formada por 376 maestros y profesores, pertenecientes a enseñanza infantil $(\mathrm{N}=136)$, primaria $(\mathrm{N}=159)$ y secundaria $(\mathrm{N}=71)$, tanto de género masculino $(\mathrm{N}=110)$ como femenino $(\mathrm{N}=266)$, y de centros públicos $(\mathrm{N}=269)$, concertados $(\mathrm{N}=106)$ y privados $(\mathrm{N}=1)$ de la Comunidad Autónoma de Extremadura. El método de selección de la muestra fue no probabilístico, incluyendo a docentes tutores de alumnos de la Universidad de Extremadura que realizaban las prácticas en diferentes centros de enseñanza extremeños. El número mínimo de participantes se determinó a partir del número de docentes de Educación Infantil, Primaria y Secundaria Obligatoria en 2019, según el Instituto de Estadística de Extremadura, considerando un error muestral del $5 \%$ y un nivel de confianza del $95.5 \%$.

\section{Creación del Cuestionario}

La creación del cuestionario de Gestión del Aprendizaje Cooperativo en el aula siguió varias fases. Así, y teniendo en cuenta que el objetivo del trabajo era crear una herramienta que permita conocer aspectos nuevos del aprendizaje cooperativo en el aula, se llevó a cabo la metodología de Carretero-Dios y Pérez (2007) para la creación de instrumentos. En primer lugar, se realizó una revisión de la literatura en fase inicial, generando una serie de ítems (15 ítems) por parte de los autores del trabajo (variados, estables, relevantes y guardar una dimensionalidad). Posteriormente, y siguiendo la Metodología Delphi, un grupo de expertos ajenos (10 investigadores) a los autores, realizaron una selección de los ítems (los 12 ítems actuales), identificando las 3 dimensiones de la escala. Más tarde, se llevó a cabo una prueba piloto, suministrando el cuestionario a varios docentes de diferentes niveles educativos (infantil, primaria y universidad). Una vez realizada la prueba piloto, se desarrolló una escala depurada, discutiendo expresiones o palabras a diferentes contextos culturales. Finalmente, se desarrolló la evaluación de la escala definitiva con los 376 maestros y profesores que se muestran en esta investigación.

\section{Procedimiento}

En primer lugar, el estudio recibió el consentimiento del Comité Ético de la Universidad de Extremadura. De esta manera, todos los participantes fueron tratados en condiciones de igualdad siguiendo la guía ética de la Asociación Americana de Psicología con respecto al consentimiento, confidencialidad y anonimato en las respuestas.

En segundo lugar, y previo al inicio de la investigación, se informó a los participantes de la voluntariedad de la misma, al igual que del tratamiento confidencial y únicamente con carácter científico que se le iba a dar a los datos. Con respecto al proceso de recogida de datos, se realizó un protocolo de actuación con la finalidad de que fuera similar durante todo el proceso. De esta manera, el investigador principal contactó con los maestros y profesores de los diferentes centros educativos objeto de estudio. Estos profesores eran aquellos docentes que eran tutores de los alumnos de infantil, primaria y secundaria que realizaban las prácticas en los diferentes centros de enseñanza (ya que la investigación se desarrolló durante el segundo semestre de curso universitario, coincidente con el inicio de las prácticas del alumnado en diferentes centros para cursar sus prácticas. Para ello, se facilitó un escrito informativo sobre los objetivos de la investigación, así como un consentimiento informado a cada participante. Una vez aceptado por parte de los profesores, se facilitó al alumnado los cuestionarios que debían rellenar.

La administración de la escala se llevó a cabo en horario escolar, ofreciendo las instrucciones pertinentes a los maestros y profesores mediante una pequeña guía, así como la información que facilitaba personalmente el alumnado en prácticas. 
Se insistió en el carácter anónimo de la investigación. Los cuestionarios fueron completados de manera individual y en un clima adecuado, que les permitía concentrarse sin tener ningún tipo de distracción. El proceso de realización de los cuestionarios duró aproximadamente 15 minutos.

\section{Análisis de los datos}

En primer lugar, mediante la prueba de Kolmogorov-Smirnov, se contrasta el supuesto de normalidad univariada de los ítems, encontrando valores no significativos ( $p>.05)$. A continuación, se llevó a cabo un análisis de las propiedades psicométricas de la escala de gestión de la cooperación en el aula, utilizando el análisis factorial confirmatorio (AFC) mediante el programa AMOS 21. Para testar el ajuste entre el modelo teórico planteado y la matriz de datos recogida se utilizaron diferentes índices: $\chi^{2} / \mathrm{gl}$ (Chi-Square / degrees of freedom), CFI (Comparative Fit Index), TLI (Tucker Lewis Index), RMSEA (Root Mean Square Error of Approximation) y SRMR (Standardized Root Mean Residual). Mediante el cálculo de la varianza media extractada y la fiabilidad compuesta se evaluó la consistencia interna de la adaptación del EGCA (Escala de Gestión de Cooperación en el Aula). Además, se realiza un análisis de validez nomológica entre los factores del EGCA y el PECDA (Escala de compromiso profesional y aspiraciones de desarrollo profesional), a través de un análisis de correlaciones bivariadas con el coeficiente de Pearson y un análisis multivariado (ANOVA) para conocer las diferencias entre grupos, utilizando para ello el programa SPSS 21.

\section{Instrumentos}

Escala de gestión de cooperación en el aula. La escala de gestión de cooperación en el aula está formada por 12 ítems que mediante una escala Likert de 5 puntos con respuestas que varían desde 1 (Totalmente desacuerdo) hasta 5 (Totalmente de acuerdo), y evalúa la planificación que hace el docente de las actividades cooperativas en el aula, mediante el diseño, seguimiento y evaluación de las actividades y tareas que se plantean en el aula. La escala se compone de tres factores: F1 Diseño (4 ítems, eg.
"Diseño y planifico las técnicas de aprendizaje cooperativo antes de aplicarlas al aula"), F2 Seguimiento (4 ítems, eg. "Intervengo en el equipo cuando sea necesario para que los alumnos/as comprendan mejor los contenidos de aprendizaje") F3 Evaluación (4 ítems, eg. "Evalúo el producto grupal y el trabajo individual dentro del equipo").

Escala de compromiso profesional y aspiraciones de desarrollo profesional (Watt \& Richardson, 2008). La Escala PECDA constituida por 17 ítems y valora el desarrollo profesional y compromiso del docente en el aula, incluye cuatro factores: esfuerzo previsto (4 ítems, i.e.: "¿Con qué intensidad te vas a esforzar para ser un maestro/profesor eficaz?"), persistencia planificada (4 ítems,i.e.: “¿Qué seguridad tienes de persistir en la enseñanza?"), aspiración de desarrollo profesional (5 ítems, i.e.: “¿Cuánto deseas participar en cursos para tu desarrollo profesional?"), y las aspiraciones de liderazgo (4 ítems, i.e.: "¿Cuánto deseas buscar un rol de supervisión/gestión en tu centro educativo?”). Las respuestas se realizaban en una escala tipo Likert de 5 puntos, dónde el 1 se correspondía con nada y el 5 se correspondía con extremadamente. Esta escala ya ha sido previamente adaptada y validada al contexto español, presentando adecuados índices de validez factorial y consistencia interna (Sánchez-Miguel, Amado, Mendo-Lázaro, LeónDel Barco y Leo, 2019). El alfa de Cronbach del total de la escala $(\alpha=.783)$, y de los factores 1 $(\alpha=.912), 2(\alpha=.733), 3(\alpha=.883)$ y $4(\alpha=.787)$ revelaron una adecuada consistencia interna. Además, en la consistencia interna de los constructos latentes presenta valores por encima de .50 en le varianza media extractada, y valores superiores a .70 en la fiabilidad compuesta y omega de Macdonald.

\section{Resultados}

\section{Análisis factorial confirmatorio}

El AFC pretende confirmar la estructura de los tres factores teóricos y si estos están relacionados o son independientes.

Así pues, una vez eliminados los valores atípicos (Tests for normality and outliers, AMOS), teniendo en cuenta algunos de los índices de ajuste 
Tabla 1. Índices de bondad de ajuste de los modelos propuestos

\begin{tabular}{|c|c|c|c|c|c|c|c|c|}
\hline \multicolumn{2}{|c|}{ Modelos } & \multirow{2}{*}{$\begin{array}{c}\text { CMIN } \\
171.366\end{array}$} & \multirow{2}{*}{$\begin{array}{c}p \\
<.001\end{array}$} & \multirow{2}{*}{$\begin{array}{c}\text { CMIN/DF } \\
3.427\end{array}$} & \multirow{2}{*}{$\begin{array}{r}\text { CFI } \\
.951\end{array}$} & \multirow{2}{*}{$\begin{array}{r}\text { TLI } \\
.935\end{array}$} & \multirow{2}{*}{$\begin{array}{c}\text { RMSEA } \\
.060\end{array}$} & \multirow{2}{*}{$\begin{array}{r}\text { SRMR } \\
.039\end{array}$} \\
\hline M1 & 3 factores relacionados & & & & & & & \\
\hline M2 & 3 factores independientes & 929.477 & $<.001$ & 17.537 & .644 & .557 & .234 & .444 \\
\hline M3 & 1 factor único & 241.172 & $<.001$ & 4.466 & .924 & .907 & .107 & .047 \\
\hline M4 & Bifactor & 105.950 & $<.001$ & 2.717 & .973 & 954 & .045 & - \\
\hline
\end{tabular}

Nota. CMIN=razón de chi-cuadrado sobre los grados de libertad; CFI=índice de ajuste comparativo; TLI=índice de

Tucker-Lewis; RMSEA=error cuadrático medio de aproximación; SRMR=raíz cuadrada media residual estandarizada.

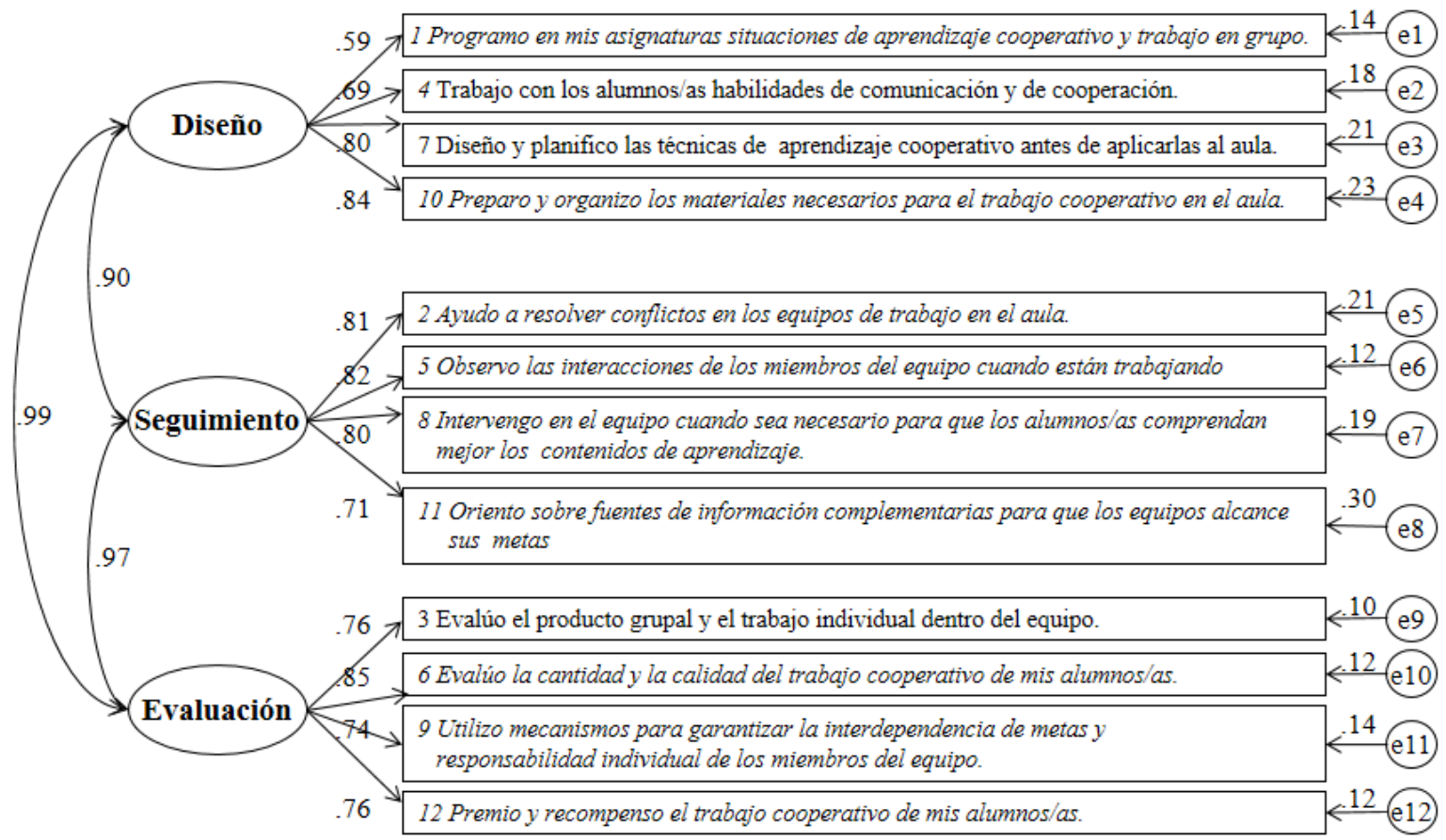

Figura 1. Modelo de tres factores relacionados de la "Escala de Gestión de la Cooperación en el Aula" (EGCA)

más utilizados $(\chi 2, \chi 2 /$ g.1, CFI, TLI, RMSEA y SRMR), mediante el método de máxima verosimilitud, se ponen a prueba cinco modelos (M): M1: tres factores relacionados; M2 tres factores independientes; M3: un solo factor; M4 modelo Bifactor. El $\chi 2$ debe adquirir valores no significativos ( $p>.05)$ (Jöreskog \& Sörbom, 1989), aunque es aceptable cuando el $\chi 2 / g .1$ es inferior a 5 (Bentler, 1989). Valores superiores a .90 de los índices incrementales (CFI y TLI) y menores o iguales a .08 del RMSEA (Browne \& Cudeck, 1993) y el SRMR (Hu \& Bentler, 1999) se consideran aceptables (Hu \& Bentler, 1995).

$\mathrm{Si}$ bien todos los modelos presentan un valor de chi cuadrado significativo $(p<.05)$. Los índices CFI, TLI de los modelos 1, 3 y 4 presentan valores superiores a .90 , siendo los modelos 1 y 4 los que presenta un mejor ajuste, con valores de $\chi 2 / \mathrm{g} .1$, inferiores, índices de ajuste CFI y TLI mayores y un valor de los indicadores RMSEA y SRMR menores a .06 (Tabla 1).

Los valores de t (rango de 10.53 a 16.18) de los coeficientes de regresión no estandarizados del modelo 1 son estadísticamente significativos. El rango de los coeficientes estandarizados del factor uno (.588-.842), dos (.711-.815) y tres (.741$.854)$ muestra que los indicadores son consistentes para la medida de los constructos, estando estos altamente relacionados $(\geq .90)$ (Figura 1).

Al mismo tiempo, se aplica el método bootstrap, utilizando un total de 1000 muestras con un intervalo de confianza al 95\% (Tabla 2). Este método permite crear un gran número de muestras con reposición de los mismos datos, comprobando así, que los valores obtenidos de las cargas factoriales, no están condicionados por la selección de la muestra. En la Tabla 2, se observa que los valores de las cargas factoriales son muy 
Tabla 2. Método Bootsrap, 1000 muestras con un IC al 95\%

\begin{tabular}{|c|c|c|c|c|c|c|}
\hline Factores & Ítems & $\begin{array}{c}\text { Cargas } \\
\text { Factoriales } \\
\end{array}$ & $\begin{array}{c}\text { Media } \\
1000 \text { muestras }\end{array}$ & $\begin{array}{l}\text { Límite } \\
\text { Inferior }\end{array}$ & $\begin{array}{c}\text { Límite } \\
\text { Superior }\end{array}$ & $p$ \\
\hline \multirow{3}{*}{ Diseño } & 1 & .588 & .585 & .510 & .656 & .002 \\
\hline & 8 & .797 & .795 & .810 & .909 & .002 \\
\hline & 11 & .842 & .841 & .647 & .817 & .002 \\
\hline \multirow{2}{*}{ Seguimiento } & 7 & .795 & .793 & .735 & .844 & .002 \\
\hline & 12 & .711 & .712 & .651 & .769 & .002 \\
\hline \multirow{2}{*}{ Evaluación } & 3 & .758 & .757 & .698 & .807 & .002 \\
\hline & 5 & .854 & .854 & .822 & .882 & .002 \\
\hline
\end{tabular}

Tabla 3. Matriz de correlaciones entre ítems

\begin{tabular}{|c|c|c|c|c|c|c|c|c|c|c|c|c|}
\hline Îtems & 1 & 2 & 3 & 4 & 5 & 6 & 7 & 8 & 9 & 10 & 11 & 12 \\
\hline CGCA 1 & 1 & & & & & & & & & & & \\
\hline CGCA 2 & $.430^{* * *}$ & 1 & & & & & & & & & & \\
\hline CGCA 3 & $.521^{* * *}$ & $.586^{* *}$ & 1 & & & & & & & & & \\
\hline CGCA 4 & $.616^{* *}$ & $.537^{* *}$ & $.579^{* *}$ & 1 & & & & & & & & \\
\hline CGCA 5 & $.472^{* * *}$ & $.675^{* *}$ & $.632^{* *}$ & $.570^{* * *}$ & 1 & & & & & & & \\
\hline CGCA 6 & $.491^{* * *}$ & $.719^{* *}$ & $.669^{* *}$ & $.594^{* * *}$ & $.719^{* * *}$ & 1 & & & & & & \\
\hline CGCA 7 & $.489^{* *}$ & $.546^{* *}$ & $.577^{* *}$ & $.518^{* *}$ & $.538^{* *}$ & $.638^{* *}$ & 1 & & & & & \\
\hline CGCA 8 & $.365^{* *}$ & $.693^{* *}$ & $.563^{* *}$ & $.465^{\text {** }}$ & $.666^{* *}$ & $.636^{* *}$ & $.547^{\text {** }}$ & 1 & & & & \\
\hline CGCA 9 & $.421^{* *}$ & $.520^{* *}$ & $.588^{* *}$ & $.487^{* *}$ & $.551^{* * *}$ & $.604^{* *}$ & $.682^{* *}$ & $.515^{* *}$ & 1 & & & \\
\hline CGCA 10 & $.449^{* *}$ & $.649^{* *}$ & $.583^{* *}$ & $.568^{* *}$ & $.569^{* *}$ & $.699^{* * *}$ & $.699^{* * *}$ & $.605^{* *}$ & $.628^{* * *}$ & 1 & & \\
\hline CGCA 11 & $.368^{* * *}$ & $.524^{* *}$ & $.529^{* *}$ & $.465^{* *}$ & $.510^{* *}$ & $.588^{* *}$ & $.582^{* *}$ & $.566^{* *}$ & $.587^{* *}$ & $.631^{* *}$ & 1 & \\
\hline CGCA 12 & $.467^{* * *}$ & $.522^{* * *}$ & $.561^{* *}$ & $.513^{* * *}$ & $.594^{* * *}$ & $.639^{* * *}$ & $.575^{* *}$ & $.596^{* *}$ & $.579^{* *}$ & $.658^{* * *}$ & $.635^{\text {** }}$ & 1 \\
\hline
\end{tabular}

similares a los valores encontrados en el análisis confirmatorio y se encuentran entre los límites inferiores y superiores del IC al 95\%, siendo, por tanto, todos ellos significativos ( $p=.002)$.

La alta correlación entre factores en el modelo de tres factores relacionados, así como correlaciones superiores a $\mathrm{r} .360$ entre los ítems (Tabla 3), sugiere la unidimensionalidad del modelo.

Los valores de t (rango de 8.7 a 10.8) de los coeficientes de regresión no estandarizados del modelo Bifactor son estadísticamente significativos. El rango de los coeficientes estandarizados (.612-.842) muestra que los indicadores son consistentes para la medida de la gestión de la cooperación en el aula como un constructo esencialmente unidimensional pero formado por tres factores específicos (Figura 2).

Asimismo, los coeficientes de varianza media extraída (VME) y fiabilidad compuesta (FC) muestran suficientes evidencias de fiabilidad ( $\mathrm{F} 1$ [4 ítems]: $\mathrm{VME}=.532 . \mathrm{FC}=.817$; F2 [4 ítems]: VME=.519, FC =.812; F3 [4 ítems]: $\mathrm{VME}=.590, \mathrm{FC}=.852$; global de la escala [12 ítems]: VME=.547, FC=.935) (Moral de la Rubia, 2019; Rodriguez, Reise, \& Haviland, 2016).

\section{Validez nomológica}

La validez nomológica se refiere al grado en que se pueden comprobar empíricamente las relaciones que un constructo puede mantener con otros que forman parte total o parcialmente de alguna teoría o teorías (Wilson, Spence, \& Kavanagh, 1989), es decir, si existe correspondencia entre la configuración teórica de los datos obtenidos y las predicciones teóricas sobre dicha configuración.

En este caso se ha relacionado las puntuaciones de la EGCA, con los factores de la Escala de Compromiso profesional y aspiraciones de desarrollo profesional (PECDA) (Watt \& Richardson, 2008). Encontrando correlaciones lineales directas significativas $(p<.001)$ entre los factores de la EGCA y el factor 1 (Esfuerzo previsto), 2 (Persistencia planificada) y 3 (Aspiraciones de desarrollo profesional) de la escala PECDA (Tabla 4). 


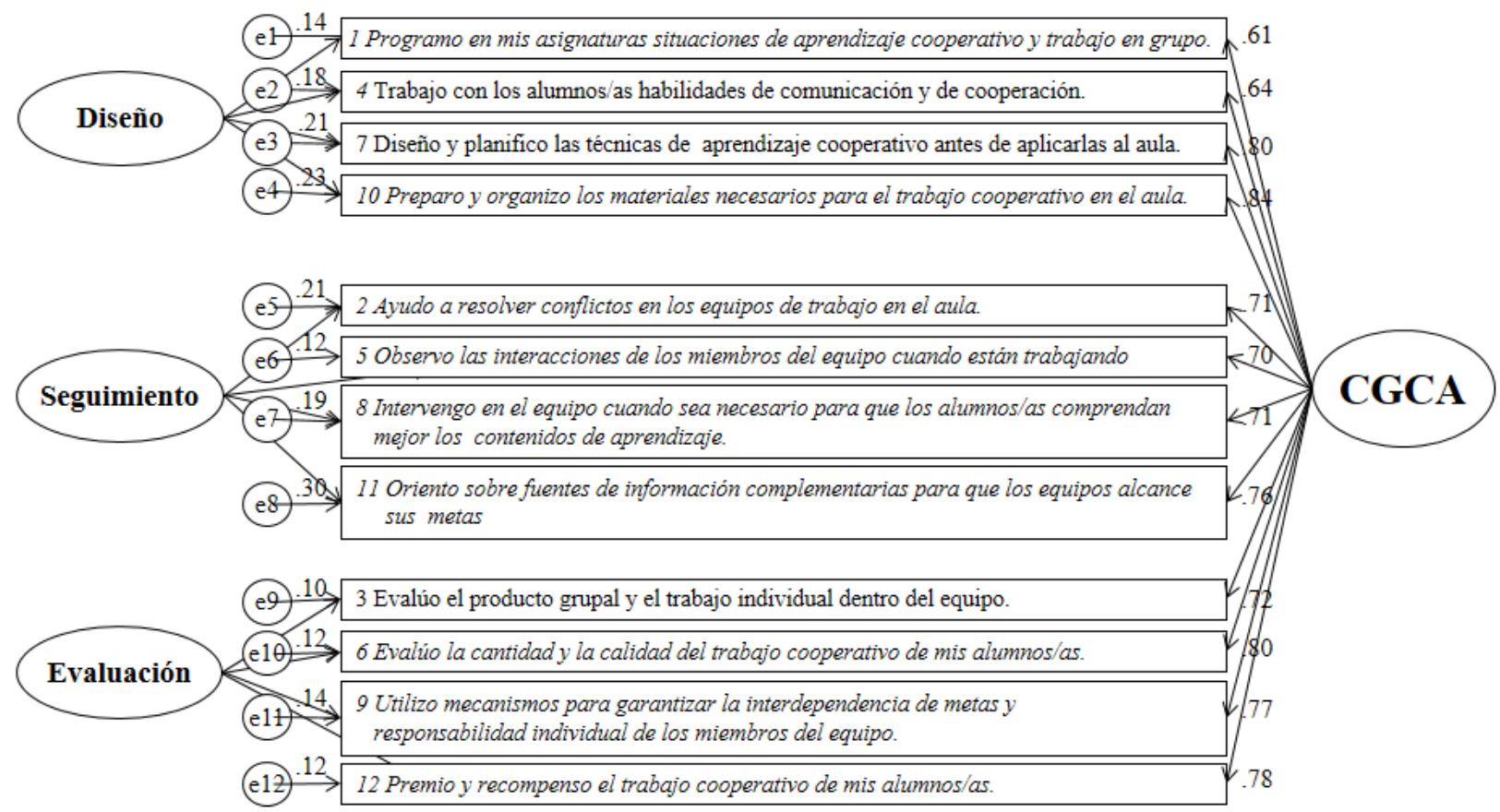

Figura 2. Modelo Bifactor de la "Escala de Gestión de la Cooperación en el Aula" (EGCA)

Tabla 4. Correlación de Pearson de los factores de las escalas PECDA/EGCA

\begin{tabular}{lcccc}
\hline & \multicolumn{4}{c}{ Factores PECDA } \\
\hline Factores EGCA & $\mathrm{F} 1$ & $\mathrm{~F} 2$ & $\mathrm{~F} 3$ & $\mathrm{~F} 4$ \\
Diseño & $.336^{* *}$ & $.152^{* *}$ & $.176^{* * *}$ & -.003 \\
Seguimiento & $.423^{* *}$ & $.230^{* *}$ & $.243^{* *}$ & -.035 \\
Evaluación & $.435^{* *}$ & $.196^{* *}$ & $.279^{* *}$ & .012 \\
\hline
\end{tabular}

Factores PECDA: F1=Esfuerzo previsto; F2=Persistencia planificada; F3=Aspiraciones de desarrollo profesional; F4=Aspiraciones de liderazgo. $* *=p<.001$

Tabla 5. Comparación de medias de la escala PECDA y los grupos de la EGCA

\begin{tabular}{|c|c|c|c|c|c|c|c|c|c|}
\hline \multirow[b]{3}{*}{ PECDA } & \multicolumn{6}{|c|}{ Grupos puntuación total de la EGCA } & \multirow{2}{*}{\multicolumn{3}{|c|}{ ANOVA }} \\
\hline & \multicolumn{2}{|c|}{ alta } & \multicolumn{2}{|c|}{ media } & \multicolumn{2}{|c|}{ baja } & & & \\
\hline & $M$ & $D T$ & $M$ & $D T$ & $M$ & $D T$ & $F$ & Sig. & $\eta^{2}$ \\
\hline$\overline{\mathrm{F} 1}$ & 19.66 & 1.03 & 19.19 & 1.35 & 17.82 & 2.13 & 43.830 & .000 & .175 \\
\hline $\mathrm{F} 2$ & 18.41 & 2.79 & 17.83 & 2.44 & 17.37 & 2.55 & 4.859 & .008 & .026 \\
\hline F3 & 22.24 & 2.45 & 20.87 & 2.92 & 20.03 & 3.09 & 17.929 & .000 & .093 \\
\hline F4 & 9.73 & 6.49 & 9.10 & 4.13 & 9.27 & 4.19 & .456 & .634 & .005 \\
\hline
\end{tabular}

Factores PECDA: F1=Esfuerzo previsto; F2= Persistencia planificada; F3=Aspiraciones de desarrollo profesional;

$\mathrm{F} 4=$ Aspiraciones de liderazgo.

Por último, se realiza un análisis multivariado ANOVA para contrastar si existen diferencias en las puntuaciones del PECDA entre docentes con puntuaciones altas, medias y bajas en el CGAP. Para lo cual, se dividió la muestra utilizada $(n=125)$ en tres grupos de igual tamaño (33\%) mediante un criterio de percentiles, asumiendo que el tercio superior, medio e inferior de las puntuación totales de la EGCA, corresponden a sujetos cuya gestión de la cooperación en el aula es alta, media y baja respectivamente.
El ANOVA (Tabla 5) muestra la existencia de diferencias en los factores 1 Esfuerzo previsto, 2 Persistencia planificada y 3 Aspiraciones de desarrollo profesional del PECDA. La comparación por pares de Bomferroni confirma que estas diferencias son significativas $(p \leq .05)$ entre los grupos de comparación baja y alta en los factores 1,2 y 3 , baja y media en el factor 1 y media y alta en el factor 3 . 


\section{Discusión}

El objetivo de este trabajo era construir y validar la escala de gestión de cooperación en el aula en una muestra de maestros de infantil y primaria, y profesores de enseñanza secundaria en España. La hipótesis que planteamos es que la validación de la escala de gestión y cooperación en el aula presentará una adecuada validez y fiabilidad en una muestra de maestros y profesores españoles. Además, se plantea como hipótesis la relación positiva de los constructos de la escala de aprendizaje cooperativo en el aula con la Escala de Compromiso profesional y aspiraciones de desarrollo profesional.

En primer lugar, y de acuerdo a esta hipótesis, los resultados de los análisis psicométricos y factoriales confirmatorios realizados, mostraron una adecuada estructura factorial, consistencia interna, varianza media extractada, fiabilidad compuesta, validez nomológica y validez concurrente del instrumento. Además, el instrumento se muestra invariante por género. De esta manera, se indica que a partir de ahora este cuestionario se puede utilizar para valorar la gestión de cooperación en el aula entre los profesionales de la enseñanza.

En línea con lo comentado previamente, se pudo testar y mostrar que el modelo factorial es recursivo, donde no hay relaciones de causalidad entre las variables endógenas y los errores de medida no están correlacionados. Además, los análisis factoriales mostraron una elevada relación entre factores, advirtiendo de la presencia de un factor general. No obstante, tanto el modelo de tres factores relacionados, como el Bifactor, obtuvieron excelentes índices de ajuste con saturaciones por encima de .60, indicando una adecuada validez factorial (Salavera \& Usán, 2018; Varela, Ábalo, Rial, \& Braña, 2006).

Resultados similares fueron hallados previamente en otras escalas que analizaban el aprendizaje cooperativo en el aula (Atxurra et al. 2015; Fernández-Río et al. 2017; León et al. 2016; Pujolás, 2009). Así, Atxurra et al. (2015) mostraron una adecuada fiabilidad y validez en la escala de aplicación del aprendizaje cooperativo (CLAS) en el contexto educativo. Por otra parte, y consistente con nuestros resultados, FernándezRío et al. (2017) obtuvieron un instrumento para valorar el aprendizaje cooperativo en el aula con valores de varianza media extractada y fiabilidad compuesta por encima de $\quad \begin{array}{llll}.50 & \text { y } & .70\end{array}$ respectivamente. Además, León et al. (2017) en una muestra de 375 estudiantes universitarios en la validación del cuestionario de potencia de equipos de aprendizaje hallaron cargas factoriales similares a los encontrados en nuestro estudio en los tres factores que conforman el cuestionario.

Respecto a la fiabilidad del CGCA, los valores de fiabilidad compuesta y varianza media extractada son considerados aceptables, teniendo valores por encima de .50 en ambos casos. Además, y tal y como se puede observar en los resultados, el modelo se muestra invariante por género, lo que refuerza la equivalencia de los constructos en ambos géneros de maestros y profesores, en la línea con estudios realizados previamente (Atxurra et al., 2015).

Por lo tanto, este estudio permite elaborar un instrumento válido y fiable para medir la gestión de la cooperación en el aula, lo cual supone una gran novedad y avance, ya que hasta el momento no había ningún instrumento que permitiera valorarlo en el profesorado. Así, gracias a los resultados de esta investigación, podemos disponer de un instrumento útil, fiable y eficaz en contextos educativos, con el objetivo de poder conocer la capacidad que tiene el docente de poder gestionar el aprendizaje cooperativo en el aula, y mostrar de manera más fidedigna los beneficios que conlleva en el alumnado (Hattie, 2008; Legrain et al. 2018).

Por otro lado, y atendiendo a la hipótesis que sugería una relación positiva de los constructos de la escala de aprendizaje cooperativo en el aula con la escala de compromiso profesional y aspiraciones de desarrollo profesional, se muestra cómo existe una relación clara con los constructos del PECDA, exaltando las relaciones mostradas entre el diseño y la evaluación de las acciones cooperativas por parte del docente con el esfuerzo y persistencia planificada. Asimismo, el seguimiento de las estrategias cooperativas que realiza el docente, se muestra en la línea de relaciones con los constructos del PECDA, lo que nos indica la relación entre las acciones planificadas del docente y su esfuerzo con el mayor desarrollo de estrategias cooperativas en el aula, que conlleva a acciones adaptativas como la 
satisfacción, implicación, apoyo hacia el alumnado... (Coldwell, 2017). Además, supondría una mejora en la iniciativa personal del alumnado, mejorando entre otros aspectos el rendimiento académico (Gorostiaga et al., 2018). De esta manera, la validez nomológica de la escala se comprueba, confirmando esta segunda hipótesis.

Como conclusiones del trabajo se muestra la creación y validación de un instrumento novedoso para valorar la gestión de la cooperación en el aula desde la perspectiva del profesorado, ya que la mayoría de trabajos habían tratado el aprendizaje cooperativo solamente desde el punto de vista del alumnado. Así, este estudio permite dotar de una herramienta válida y eficaz para el contexto educativo que permita analizar una acción docente tan relevante como es el aprendizaje cooperativo.

En cuanto a las limitaciones del estudio se encuentran la única medición transversal de los maestros y profesores, pudiendo cotejar de forma conjunta la valoración transversal con la observación directa en el aula. Asimismo, y en relación con esta limitación, se destaca que solamente se ha realizado una valoración mediante la percepción que tiene el profesorado de la gestión de la cooperación en el aula, pudiendo llevar a cabo un análisis in vivo de los tipos de gestión que desarrollan los docentes. En línea con el trabajo de Veenman, Veenman, Benthum, Bootsma, Dieren, y Kemp (2002), quiénes diseñaron un instrumento para analizar el aprendizaje cooperativo desde una perspectiva externa, no desde la propia percepción de los participantes.

De la misma manera, tampoco se ha valorado la estabilidad temporal del cuestionario, por lo que en futuros trabajos se recomienda realizar varias valoraciones en momentos diferentes del curso académico. Además, y con el objetivo de mejorar la calidad del instrumento, la valoración conjunta a docentes y alumnos sería una prospectiva de futuro a desarrollar, la cual mejoraría y dotaría de mayor calidad al instrumento científico. Asimismo, ampliar la selección de la muestra al contexto universitario y al contexto de enseñanza privada (universitaria y no universitaria), mejoraría la generalización del trabajo. Finalmente, y como última prospectiva de futuro, el análisis mediante modelos mixtos (cualitativos y cuantitativos), nos permitiría conocer en mayor profundidad las consecuencias que conlleva la aplicación del AC en las aulas.

\section{Referencias}

Atxurra, C., Villardón-Gallego, L., \& Calvete, E. (2015). Diseño y validación de la escala de aplicación del aprendizaje cooperativo (CLAS). Revista de Psicodidáctica, 20(2), 339-357. doi:10.1387/RevPsicodidact.11917

Bentler, P. M. (1989). EQS structural equations program manual. Los Angeles, CA: BMDP Statistical Software.

Biggs, J., \& Tang, C. (2011). Teaching for quality learning at University: What the student does. Maidenhead: M cGraw-Hill. Open University Press.

Browne, M. W., \& Cudeck, R. (1993). Alternative ways of assessing model fit. In: K. A. Bollen \& J. S. Long (Eds.), Testing structural equation models (pp. 136-162). Beverly Hills, CA: Sage.

Caena, F. (2014). Initial teacher education in Europe: An overview of policy issues. Brussels: European Commission.

Cavanagh, M. (2011). Students' experiences of active engagement through cooperative learning activities in lectures. Active Learning in Higher Education, 12(1), 23-33. doi:10.1177/1469787410387724

Carretero-Dios, H., \& Pérez, C. (2007). Normas para el desarrollo y revisión de estudios instrumentales: Consideraciones sobre la Elección de tests en la investigación psicológica. International Journal of Clinical and Health Psychology, 7(3), 863-82.

Coldwell, M. (2017). Exploring the influence of professional development on teacher careers: A path model approach. Teaching and Teacher Education, 61, 189-198.

Dat-Tran, V. (2014). The effects of cooperative learning on the academic achievement and knowledge retention. International Journal of Higher Education, 3(2), 131-140. doi:org/10.5430/ijhe.v3n2p131

Deutsch, M. (1949). A theory of competition. Human Relations, 2, 129-151.

Díaz-Aguado, M. J. (2003). Educación interculural y aprendizaje cooperativo. Madrid: Pirámide. 
Dyson, B., \& Casey, A. (2016). Cooperative Learning in Physical Education and Physical Activity. A practical introduction. London: Roughtledge.

Fernández-Río, J., Cecchini, J. A., MéndezGiménez, A., Méndez-Alonso, D., \& Prieto, J.A. (2017). Design and validation of a questionnaire to assess cooperative learning in educational contexts. Anales de Psicología, 33(3), 680-688.

doi.org/10.6018/analesps.33.3.251321

Gillies, R., \& Boyle, M. (2006). Ten australian elementary teachers' discourse and reported pedagogical practices during cooperative learning. The Elementary School Journal, 106(5), 429-452. doi:10.1086/505439

Gillies, R., \& Boyle, M. (2010). Teachers' reflections on cooperative learning: Issues of implementation. Teaching and Teacher Education, 26(4), 933-940. doi:10.1016/j.tate.2009.10.034

Gorostiaga, A., Balluerka, N., Ulacia, I., \& Aliri, J. (2018). Evaluación de la iniciativa personal en el ámbito educativo y su relación con la actitud emprendedora y el rendimiento académico. Revista Iberoamericana de Diagnóstico y Evaluación - e Avaliação Psicológica, 48(3), 105-116.

Grapentine, T. (1995). Problematic scales: When measuring quality, expectations scales exhibir several drawbacks. Marketing Research, 6(4), 8-12.

Hattie, J. (2008). Visible learning: A synthesis of over 800 meta-analyses relating to achievement. London: Routledge.

Hu, L., \& Bentler, P. M. (1995). Evaluating model fit. In R. Hoyle (Ed.), Structural equation modeling: Issues, concepts, and applications (pp. 76-99). Newbury Park, CA: Sage.

Hu, L., \& Bentler, P. M. (1999). Cutoff criteria for fit indexes in covariance structure analysis: Conventional criteria versus new alternatives. Structural Equation Modeling, 6, 1-55.

Johnson, D. W. (2009). Reaching out: Interpersonal effectiveness and selfactualization, Boston: Allyn \& Bacon.

Johnson, D. W., \& Johnson, R. T (1983). Social interdependence and perceives academic and personal support in the classroom. The Journal of Psychology, 120, 77-82.
Johnson, D. W., \& Johnson, R. T. (2008). Social Interdependence Theory and cooperative learning: The teacher's role. In Gillies, R. M., Ashman, A. \& Terwel, J. (Eds.). Teacher's Role in Implementing Cooperative Learning in the Classroom (pp.9-37).New York, U.S.A: Springer. doi.org/10.1007/978-0-387-70892-8_1

Johnson, D. W., \& Johnson, R. (2005). New developments in Social Interdependence Theory. Genetic, Social, \& General Psychology Monographs, 131(4), 285-358. doi.org/10.3200/MONO.131.4.285-358

Johnson, D. W., \& Johnson, R. T. (2009). An educational psychology success story: Social Interdependence Theory and cooperative learning. Educational Researcher, 38(5), 365379. doi.org/10.3102/0013189X09339057

Jöreskog, K. G., \& Sörbom, D. (1989). Lisrel 7. A guide to the program and applications. (2nd Ed.) Chicago, Illinois: SPSS Inc.

Kaiser, H. F. (1960). The application of electronic computers to factor analysis. Educational and Psychological Measurement, 20, 141-151. doi.org/10.1177/001316446002000116

Kelley, H. H., \& Thibaut, J. W. (1969). Group problem solving. En G. Linzey y E. Aronson (Eds.), Handbook of social psychology (pp. 41-101). Reading, Mass: Addison-Wesley.

Legrain, P., Escalié, G., Lafont, L., \& Chaliès, S. (2018). Cooperative learning: A relevant instructional model for physical education pre-service teacher training?. Physical Education and Sport Pedagogy, 24(1), 73-86. doi.org/10.1080/17408989.2018.1561838

León, B., Felipe, E., Iglesias, D., \& Latas, C. (2011). El aprendizaje cooperativo en la formación inicial del profesorado de Educación Secundaria. Revista de Educación, 354, 715-729.

León, B., Felipe, E., Iglesias, D., \& Marugán, M. (2014). Determinantes en la eficacia del aprendizaje cooperativo. Una experiencia en el EEES. Revista de Investigación Educativa, $32(2)$, 411-424. doi.org/10.6018/rie.32.2.172721

León, B., Mendo, S., Felipe, E., Polo, M. I., \& Fajardo, F. (2017). Team potency and cooperative learning in the university setting. Journal of Psychodidactics, 21(2), 9-15. 
doi:10.1387/RevPsicodidact.14213.

León del Barco, B., \& Latas-Pérez, C. (2007). La formación en técnicas de aprendizaje cooperativo del profesor universitario en el contexto de la convergencia europea. Revista de Psicodidáctica,12(2), 269-278. doi:10.1387/RevPsicodidact.224

Moral-de la Rubia, J. (2019). Revisión de los criterios para validez convergente estimada a través de la varianza media extraída. Psychologia, 13(2), 25-41. doi:10.21500/19002386.4119.

Monereo, C., Castelló, M., \& MartínezFernández, R. (2013). Predicción del éxito en el trabajo en equipo de estudiantes de Secundaria. Revista de Psicodidáctica, 18(2), 235-255. doi:10.1387/RevPsicodidact.6776

Muñiz, J., Elosua, P., \& Hambleton, R. (2013). Directrices para la traducción y adaptación de los test: Segunda edición. Psicothema, 25(2), 151-157. doi:10.7334/psicothema2013.24

OECD (2015). PISA 2015 Assessment and analytical framework: Science, reading, mathematic, financial literacy and collaborative problem solving. Paris: OCDE. doi:10.1787/9789264281820-8-en

Pujolás, P. (2009). La calidad en los equipos de aprendizaje cooperativo: Algunas consideraciones para el cálculo del grado de cooperatividad. Revista de Educación, 349, 225-239.

Rodriguez, A., Reise, S. P., \& Haviland, M. G. (2016). Applying bifactor statistical indices in the evaluation of psychological measures. Journal of Personality Assessment, 98(3), 223237. doi:10.1080/00223891.2015.1089249

Salavera, C., \& Usán, P. (2018). Adaptación del Cuestionario de Competencia Interpersonal ICQ-15 con Población Adolescente Hispanohablante. Revista Iberoamericana de Diagnóstico y Evaluación - e Avaliação Psicológica, $\quad 48(3), \quad$ 29-39. https://doi.org/10.21865/RIDEP48.3.03

Sánchez-Miguel, P. A., Amado, D., MendoLázaro, S., León-Del Barco, B., \& Leo, F. M. (2019). Adaptación y validación al español de la Escala de Compromiso Profesional y Aspiraciones de Desarrollo Profesional. Revista Iberoamericana de Diagnóstico y Evaluación - e Avaliação Psicológica, 3(52),
181-191.

https://doi.org/10.21865/RIDEP52.3.14

Slavin, R. E. (2011). Instruction based on cooperative learning. In R. E. Mayer \& P. A. Alexander (Eds.), Handbook of Research on Learning and Instruction (pp. 344-360). New York: Taylor \& Francis.

Spittle, M., Jackson, K., \& Casey, M. (2009). Applying self-determination theory to understand the motivation for becoming a physical education teacher. Teaching and Teacher Education, 25(1), 190-197.

Varela, J., Abalo, J., Rial, A., \& Braña, T. (2006). El análisis factorial confirmatorio de segundo nivel. En J.P. Lévy y J. Varela (Eds.), Modelización con Estructuras de Covarianzas en Ciencias Sociales (pp.239-258). A Coruña: Netbiblo.

Veenman, S., Benthum, N., Bootsma, D., Dieren, J., \& Kemp, N. (2002). Cooperative learning and teacher education. Teaching and Teacher Education, 18(1), 87-103. doi:10.1016/S0742-051X(01)00052-X

Yi, Z., \& LuXi, Z. (2012). Implementing a cooperative learning. Educational studies, 38(2), 165-173. doi.org/10.1080/03055698.2011.598687

Watt, H. M. G., \& Richardson, P. W. (2008). Motivations, perceptions, and aspirations concerning teaching as a career for different types of beginning teachers. Learning and Instruction, 18, 408-428.

Wilson, P. H., Spence, S. H., \& Kavanagh, D. J. (1989). Cognitive behavioural interviewing for adult disorders: A practical handbook. Londres: Routledge. 


\section{Anexo}

Nombre y Apellidos:

Género: Masculino Femenino

Centro Educativo:

Nivel de Enseñanza: E.Infantil E. Primaria E. Secundaria

Especialidad.

Localidad:

Situación Profesional: Funcionario Interino con vacante Interino sin vacante Años de Experiencia:

Tipo de centro: Público

Concertado

Privado

\begin{tabular}{|c|c|c|c|c|c|}
\hline $\begin{array}{l}\text { xodee con un círculo el número que mejor indique la frecuencia con la que lleva a cabo la } \\
\text { xponen a continuación. } 1 \text { : Nunca; } 2 \text { : Casi Nunca; 3: Algunas veces; 4: Casi Siempre; 5: S } \\
\text { lases... }\end{array}$ & & & & & \\
\hline 1. Programo en mis asignaturas situaciones de aprendizaje cooperativo y trabajo en grupo. & 1 & 2 & 3 & 4 & 5 \\
\hline 2. Trabajo con los alumnos/as habilidades de comunicación y de cooperación. & 1 & 2 & 3 & 4 & 5 \\
\hline 3. Evalúo el producto grupal y el trabajo individual dentro del equipo. & 1 & 2 & 3 & 4 & 5 \\
\hline 4. Ayudo a resolver conflictos en los equipos de trabajo en el aula. & 1 & 2 & 3 & 4 & 5 \\
\hline 5. Evalúo la cantidad y la calidad del trabajo cooperativo de mis alumnos/as. & 1 & 2 & 3 & 4 & 5 \\
\hline 6. Observo las interacciones de los miembros del equipo cuando están trabajando & 1 & 2 & 3 & 4 & 5 \\
\hline $\begin{array}{l}\text { 7. Intervengo en el equipo cuando sea necesario para que los alumnos/as comprendan mejor } \\
\text { los contenidos } \\
\text { de aprendizaje. }\end{array}$ & 1 & 2 & 3 & 4 & 5 \\
\hline 8. Diseño y planifico las técnicas de aprendizaje cooperativo antes de aplicarlas al aula. & 1 & 2 & 3 & 4 & 5 \\
\hline $\begin{array}{l}\text { 9. Utilizo mecanismos para garantizar la interdependencia de metas y responsabilidad } \\
\text { individual de los } \\
\text { miembros del equipo. }\end{array}$ & 1 & 2 & 3 & 4 & 5 \\
\hline 10. Premio y recompenso el trabajo cooperativo de mis alumnos/as. & 1 & 2 & 3 & 4 & 5 \\
\hline 11. Preparo y organizo los materiales necesarios para el trabajo cooperativo en el aula. & 1 & 2 & 3 & 4 & 5 \\
\hline $\begin{array}{l}\text { 12.Oriento sobre fuentes de información complementarias para que el equipo alcance sus } \\
\text { metas }\end{array}$ & 1 & 2 & 3 & 4 & 5 \\
\hline
\end{tabular}

